\title{
Does Retailer Power Lead to Exclusion?
}

\author{
Patrick Rey and Michael D. Whinston ${ }^{1}$
}

February 2011

${ }^{1}$ Rey: Toulouse School of Economics (GREMAQ and IDEI), prey@cict.fr; Whinston: Department of Economics, Northwestern University and NBER, mwhinston@northwestern.edu. Whinston thanks the NSF and the Toulouse Network for Information Technology for financial support. 


\begin{abstract}
This paper examines whether retailer bargaining power and upfront slotting allowances prevent small manufacturers (who have no bargaining power) from obtaining adequate distribution. In contrast to the findings of Marx and Shaffer (2007), who showed that all equilibria involve limited distribution (i.e., exclusion of a retailer), we show that there is always an equilibrium in which full distribution is obtained, provided that full distribution is the industry profit-maximizing outcome. The key feature leading to this differing result is that we do not restrict each retailer to offering the manufacturer a single tariff.
\end{abstract}




\section{Introduction}

In a recent paper, Marx and Shaffer (2007) study a model of vertical contracting between a manufacturer and two retailers in which bargaining power resides with the retailers, who simultaneously make take-it-orleave-it offers to the manufacturer. They reach the striking conclusion that when retailers have such bargaining power, all equilibria lead to exclusion of a retailer from carrying the manufacturer's product. This is so even though, following the reasoning of Segal (1999), exclusion would not occur in their model were it instead the manufacturer who made (public) take-it-or-leave it offers to the retailers. Marx and Shaffer's exclusion equilibria are sustained with "three-part tariffs" (described below in more detail) in which the manufacturer pays the retailer an upfront "slotting fee", and then the retailer can buy the manufacturer's product under two-part tariff pricing terms. Based on their findings, they conclude that upfront slotting payments can prevent small manufacturers (who have no bargaining power) from obtaining adequate distribution (i.e., get carried by all retailers).

Following the Marx and Shaffer article, Miklos-Thal, Rey, and Verge (forth.) observed that the result does not hold when retailers can make contingent offers, explicitly conditional on whether they have exclusivity. Miklos-Thal et al. show that in that case there is always an equilibrium in the Marx and Shaffer model that maximizes industry (or "vertical structure") profits and does not involve any exclusion. This finding, compared to the Marx and Shaffer result, seems to present a somewhat paradoxical conclusion: exclusion happens only if (explicit) exclusion is not allowed.

In this short paper we show that the key feature leading to the Marx and Shaffer result is rather Marx and Shaffer's restriction of retailers to 
offering a single three-part tariff. Specifically, we show that when retailers can offer a menu of tariffs, there is always an equilibrium in which no exclusion occurs and industry profits are maximized, even when the tariffs cannot be made explicitly conditional on exclusivity. Our conclusion indicates that neither retailer market power nor slotting fees necessarily limit the distribution of manufacturers' products. The resulting outcome may be better for consumers than Marx and Shaffer's exclusionary outcome (retail coverage availability is greater, while prices may be either higher or lower), but is generally not socially optimal because of the industry's market power.

The rest of the paper is organized as follows: In Section 2, we describe the Marx and Shaffer model and result. In Section 3, we first present our analysis and result in the context of the Marx and Shaffer model, allowing for menus of three-part tariffs. Section 4 shows that a similar result holds when we allow for more general tariffs and alternative types of arrangements.

\section{The Marx and Shaffer Model and Result}

The basic framework of Marx and Shaffer is as follows. Two differentiated retailers $R_{1}$ and $R_{2}$ distribute the product of a manufacturer $M$. Retailers incur no costs other than what they pay the manufacturer, while the manufacturer's cost of producing quantities $q_{1}$ and $q_{2}$ (where $q_{i}$ is the quantity sold to retailer $\left.i\right)$ is $c\left(q_{1}, q_{2}\right)$. Retailers have all the bargaining power in their bilateral relations with the manufacturer; their interaction is therefore modeled as follows:

1. $R_{1}$ and $R_{2}$ simultaneously make take-it-or-leave-it "three-part tariff" offers to $M$, stipulating wholesale prices $w_{1}$ and $w_{2}$ as well as lump-sum fees (more on this below). 
2. $M$ accepts or rejects each retailer's offer; accepted contracts are public.

3. The retailers with accepted tariffs compete on the downstream market and the relevant tariff conditions are implemented.

Marx and Shaffer assume that the stage 3 retail equilibrium when both retailers are making purchases at wholesale prices $w_{1}$ and $w_{2}$ results in profits for $M$ and $R_{i}$ of $\pi_{M}\left(w_{1}, w_{2}\right)$ and $\pi_{i}\left(w_{i}, w_{-i}\right)$ respectively. ${ }^{1}$ Marx and Shaffer moreover assume that $\pi_{i}\left(w_{i}, w_{-i}\right)$ is nonincreasing in $w_{i}$ and nondecreasing in $w_{-i}$, as would be expected when the retailers are (imperfect) substitutes, with these effects holding strictly at any $\left(w_{1}, w_{2}\right)$ such that $\pi_{i}\left(w_{i}, w_{-i}\right)>0$. The industry profit $\pi_{M}\left(w_{1}, w_{2}\right)+$ $\sum_{i} \pi_{i}\left(w_{i}, w_{-i}\right)$ is maximized at $\left(w_{1}, w_{2}\right)=\left(w_{1}^{*}, w_{2}^{*}\right)$, where it is equal to $\Pi^{*}$. If $R_{i}$ instead monopolizes the retail market, the joint profit of $M$ and $R_{i}$ is $\pi_{M}\left(w_{i}, \infty\right)+\pi_{i}\left(w_{i}, \infty\right){ }^{2}$ which achieves its maximum, $\Pi_{i}^{m}$, at wholesale price $w_{i}=w_{i}^{m}$. Marx and Shaffer focus on the case in which industry profits are greater when both retailers are active: $\Pi^{*}>$ $\max \left\{\Pi_{1}^{m}, \Pi_{2}^{m}\right\}$.

Finally, Marx and Shaffer assume that each retailer's offer is a "threepart tariff," in which $R_{i}$ 's payments to $M$ have the form:

$$
t_{i}\left(q_{i}\right)= \begin{cases}-S_{i} & \text { if } q_{i}=0 \\ -S_{i}+F_{i}+w_{i} q_{i} & \text { if } q_{i}>0\end{cases}
$$

Such a tariff, which we denote by $T_{i}=\left(S_{i}, F_{i}, w_{i}\right)$, thus involves:

- an upfront "slotting" payment $S_{i}$ paid by $M$ to $R_{i}$;

\footnotetext{
${ }^{1}$ The notation " $-i$ " refers to $R_{i}$ 's rival.

${ }^{2}$ There is a slight abuse of notation here, which simplifies the exposition; more formally, $M$ 's profit under exclusivity is either $\pi_{M}\left(w_{1}, \infty\right)$ or $\pi_{M}\left(\infty, w_{2}\right)$.
} 
- a conditional fee $F$, paid to $M$ by $R_{i}$, but only if $R_{i}$ actually buys a positive quantity;

- a per unit wholesale price $w_{i}$.

Marx and Shaffer show that under these conditions all pure strategy subgame perfect Nash equilibria involve exclusion: only one retailer makes purchases from the manufacturer.

To proceed, observe first that the joint payoff of the manufacturer $M$ and a retailer $R_{i}$ cannot be less than what they could achieve in an exclusive relationship:

Lemma 1 When the set of allowable contract offers includes three-part tariffs, in any subgame perfect Nash equilibrium the joint payoff of the manufacturer $M$ and a retailer $R_{i}(i=1,2)$ cannot be less than $\Pi_{i}^{m}$.

Proof. Let $\Pi_{M}$ and $\Pi_{i}$ be the equilibrium payoffs of $M$ and $R_{i}$, respectively, and suppose that $\Pi_{M}+\Pi_{i}<\Pi_{i}^{m}$. Note that $M$ must earn no more than $\Pi_{M}$ by accepting only a contract from $R_{-i}$ since otherwise $M$ would have a profitable deviation.

Now consider a deviation by $R_{i}$ in which he offers three-part tariff $\widetilde{T}=(\widetilde{S}, \widetilde{F}, \widetilde{w}) \equiv\left(\Pi_{i}^{m}-\delta-\left(\Pi_{M}+\varepsilon\right), \pi_{i}\left(w_{i}^{m}, \infty\right)-\delta, w_{i}^{m}\right)$ where $\varepsilon \in\left(0,\left(\Pi_{i}^{m}-\Pi_{M}-\Pi_{i}\right) / 2\right)$ and $\delta \in(0, \varepsilon)$. This contract faces $R_{i}$ with wholesale price $w_{i}^{m}$, together with a fixed fee for positive quantities that is lower than its monopoly revenues for that price. If $M$ accepts only $R_{i}$ 's tariff $\widetilde{T}, R_{i}$ will thus buy from $M$, and $M$ 's profit will be:

$$
\begin{aligned}
\widetilde{\Pi}_{M} & =-\widetilde{S}+\widetilde{F}+\pi_{M}\left(w_{i}^{m}, \infty\right) \\
& =\left(\Pi_{M}+\varepsilon\right)+\delta-\Pi_{i}^{m}+\pi_{i}\left(w_{i}^{m}, \infty\right)-\delta+\pi_{M}\left(w_{i}^{m}, \infty\right) \\
& =\Pi_{M}+\varepsilon,
\end{aligned}
$$


which exceeds his payoff from accepting no contract or only a contract offer from $R_{-i}$. Thus, $M$ must accept $R_{i}$ 's contract $\widetilde{T}$ in any continuation equilibrium. $R_{i}$ 's profit in any continuation equilibrium is therefore bounded below by the upfront payment he receives in tariff $\widetilde{T}$ : [I introduced an inequality sign in the equation below, reflecting the change in $\left.\varepsilon \in\left(0,\left(\Pi_{i}^{m}-\Pi_{M}-\Pi_{i}\right) / 2\right)\right]$

$$
\widetilde{S}=\left(\Pi_{i}^{m}-\Pi_{M}\right)-\varepsilon-\delta>\left(\Pi_{i}+2 \varepsilon\right)-\varepsilon-\delta=\Pi_{i}+\varepsilon-\delta>\Pi_{i} .
$$

Hence, $R_{i}$ would have a profitable deviation.

Marx and Shaffer's exclusion result follows from Lemma 1. To see this, observe that, as noted by Marx and Shaffer (see their Lemma 1), in any equilibrium in which $M$ deals with both retailers, each $R_{i}$ must make $M$ indifferent between accepting its offer and rejecting it, since $R_{i}$ could otherwise demand a slightly larger upfront payment. ${ }^{3}$ When each $R_{i}$ can offer only a single three-part tariff $T_{i}$, this implies that if in equilibrium $M$ were buying from both retailers, then $M$ 's payoff would be the same as in an exclusive relationship with $R_{i}$ under the same tariff $T_{i}$. On the other hand, if both retailers are making purchases, retailer $R_{i}$ 's payoff would have to be strictly less than his payoff in an exclusive relationship under tariff $T_{i}$ (since his profit is decreasing in the wholesale price $\left.w_{-i}\right)$. Since their joint payoff under tariff $T_{i}$ can be no greater than $\Pi_{i}^{m}$, this would imply that their joint payoff in this equilibrium would be strictly less than $\Pi_{i}^{m}$, which is impossible by Lemma 1 . So any pure

\footnotetext{
${ }^{3}$ The argument assumes here that, in case of multiple continuation equilibria, the selection among those equilibria does not depend on the upfront payment, which becomes a sunk cost at that stage.

Also, in their Lemma 1 Marx and Shaffer implicitly assume that $M$ strictly prefers accepting either or both offers to accepting neither, i.e. that $\Pi_{M}>0$. Our Assumption 1 below implies that this condition holds in any equilibrium.
} 
strategy subgame perfect Nash equilibrium must have only one retailer making purchases from $M$.

\section{Menus of Three-Part Tariffs}

Marx and Shaffer's result assumes that retailers' offers consist of a single three-part tariff. We now examine what happens when each retailer can instead offer a menu of three-part tariffs. With such a menu, at stage 2 $M$ can choose which tariff, if any, to accept from each retailer. We show that when such menus are possible a subgame perfect Nash equilibrium exists that implements the industry profit-maximizing outcome and gives each retailer $R_{i}$ a profit equal to his "contribution" to industry profit, $\Delta_{i} \equiv \Pi^{*}-\Pi_{-i}^{m} \geq 0$. The manufacturer earns the residual $\Delta_{M} \equiv \Pi^{*}-$ $\sum_{i} \Delta_{i}=\sum_{i} \Pi_{i}^{m}-\Pi^{*}$.

We first note that Lemma 1 implies that retailers cannot earn more than their contributions:

Corollary 2 When the set of allowable contract offers includes threepart tariffs, in any subgame perfect Nash equilibrium a retailer $R_{i}$ 's payoff cannot exceed his contribution, $\Delta_{i}=\Pi^{*}-\Pi_{i}^{m}$.

Proof. Since $\Pi_{M}+\Pi_{-i} \geq \Pi_{-i}^{m}$ by Lemma 1, and the total payoff is bounded above by $\Pi^{*}$, we have $\Pi_{i} \leq \Pi^{*}-\left(\Pi_{M}+\Pi_{-i}\right) \leq \Pi^{*}-\Pi_{-i}^{m}=\Delta_{i}$.

We also will make the following assumption:

Assumption 1: $\Pi_{1}^{m}+\Pi_{2}^{m}>\Pi^{*}$.

Assumption 1 captures the notion that sales through the two retailers are substitutes in either downstream demand or upstream manufacturing costs (or both). The assumption guarantees that in equilibrium $M$ 
always strictly prefers to accept one or both retailer offers rather than accept none (i.e., $\Pi_{M}>0$ ), a feature that is implicitly assumed by Marx and Shaffer (see footnote 3 ).

To proceed, we construct an equilibrium supporting the (non-exclusionary) industry profit-maximizing outcome as follows: Suppose that each retailer $i=1,2$ offers $M$ a menu $C_{i}=\left(T_{i}^{C}, T_{i}^{E}\right)$ which gives $M$ a choice between two three-part tariffs, $T_{i}^{C}=\left(S_{i}^{C}, F_{i}^{C}, w_{i}^{C}\right)$ and $T_{i}^{E}=\left(S_{i}^{E}, F_{i}^{E}, w_{i}^{E}\right)$, designed respectively for "common agency" and "exclusive dealing." (Note that since these three-part tariffs do not have any explicit exclusivity requirements, $M$ is free to accept contracts $T_{1}^{E}$ and $T_{2}^{E}$.) The two three-part tariffs have the following structure:

- both options involve an upfront payment that gives $R_{i}$ its full contribution to the industry profits: $S_{i}^{C}=S_{i}^{E}=\Delta_{i}$;

- the option designed for common agency, $T_{i}^{C}$, has $w_{i}^{C}=w_{i}^{*}$, to sustain the industry profit-maximizing outcome, and $F_{i}^{C}=\pi_{i}\left(w_{i}^{*}, w_{-i}^{*}\right)$, equal to $R_{i}$ 's equilibrium profit (gross of the payments $S_{i}$ and $F_{i}$ );

- the option designed for exclusive dealing, $T_{i}^{E}$, has $w_{i}^{E}=w_{i}^{m}$, to sustain the bilateral profit-maximizing outcome, and $F_{i}^{E}=$ $\pi_{i}\left(w_{i}^{m}, \infty\right)$, equal to the profit that $R_{i}$ can obtain under exclusivity.

In the equilibrium, $M$ accepts the two tariffs $\left(T_{1}^{C}, T_{2}^{C}\right)$, and the retailers then implement the profit-maximizing outcome: wholesale prices $\left(w_{1}^{C}, w_{2}^{C}\right)=\left(w_{1}^{*}, w_{2}^{*}\right)$ generate the industry profit-maximizing prices and quantities if both retailers buy at these prices, and they are indeed willing to buy since the conditional fees $\left(F_{1}^{C}, F_{2}^{C}\right)$ do not exceed their corresponding flow profits. In this continuation equilibrium, $M$ recovers both retailers' flow profits through the conditional fees $F_{i}^{C}$; therefore, each 
$R_{i}$ just obtains his contribution to industry profits $\left(\Delta_{i}\right)$ through the upfront payment $S_{i}$, whereas $M$ obtains the residual $\Delta_{M}=\Pi^{*}-\Delta_{1}-\Delta_{2}$. Moreover, the joint profit of $M$ and each retailer $R_{i}, \Delta_{i}+\Delta_{M}$, equals $\Pi_{i}^{m}$.

We now argue that both retailers offering these tariffs, and $M$ accepting $\left(T_{1}^{C}, T_{2}^{C}\right)$ constitute a subgame perfect Nash equilibrium. Consider first $M$ 's acceptance decision. If $M$ accepts a tariff from both retailers, in any continuation equilibrium each retailer $R_{i}$ must earn at least $S_{i}$, which equals $\Delta_{i}$. So $M$ 's profit cannot exceed $\Pi^{*}-\Delta_{1}-\Delta_{2}=\Delta_{M}$, which is what he gets by accepting $\left(T_{1}^{C}, T_{2}^{C}\right)$. If, instead, $M$ accepts only a contract from one retailer, say $R_{i}$, then once again $R_{i}$ 's payoff is at least $\Delta_{i}$. So $M$ 's profit is bounded above by $\Pi_{i}^{m}-\Delta_{i}=\Delta_{M}$. Hence, accepting $\left(T_{1}^{C}, T_{2}^{C}\right)$, which gives profit $\Delta_{M}>0$, is an optimal choice for $M$.

Now consider deviations by a retailer, say $R_{i}$. Following any deviation, $M$ must earn at least $\Delta_{M}$, since this is what she receives by accepting only retailer $R_{-i}$ 's tariff $T_{-i}^{E}$. So if $M$ accepts only a contract from $R_{i}, R_{i}$ 's profit cannot increase since the joint profit of $M$ and $R_{i}$ is bounded above by their equilibrium joint profit, $\Pi_{i}^{m}$. If $M$ instead accepts a contract from both retailers, then since $R_{-i}$ must earn at least $\Delta_{-i}\left(=S_{-i}^{C}=S_{-i}^{E}\right)$ in any continuation equilibrium, $R_{i}$ 's profit is bounded above by $\Pi^{*}-\Delta_{M}-\Delta_{-i}=\Delta_{i}$. Thus, no profitable deviation exists for the retailers either.

The above offers and contination play thus constitute a subgame perfect Nash equilibrium in which both retailers are active and each retailer $R_{i}$ earns its maximal achievable profit, $\Delta_{i}$. Both retailers therefore prefer this equilibrium to any other equilibrium. In summary:

Proposition 3 When retailers can offer menus of three-part tariffs, 
there exists a subgame perfect Nash equilibrium in which both retailers are active, industry profits are at the industry profit-maximizing level $\left(\Pi^{*}\right)$, and the retailers earn their respective contributions to these profits $\left(\Delta_{1}\right.$ and $\left.\Delta_{2}\right)$.

The menus of contracts offered by each retailer $R_{i}$ here can be thought of as allowing $M$ to respond in his contract with $R_{i}$ to information $M$ subsequently learns about the other retailer $R_{-i}$ 's offer. That this can matter for equilibria can be seen in previous work on contracting with externalities/common agency [Segal and Whinston (2003), Martimort and Stole (2003)]. In the Marx and Shaffer setting, when menus are not allowed $M$ has only an inefficient (i.e., not profit-maximizing) option for dealing exclusively with each retailer $R_{i}$. This leads retailer $R_{-i}$ to be overly aggressive in his contract offer, leaving too little surplus for $M$ and $R_{i}$, and causing $R_{i}$ to deviate in a manner that leads $M$ to deal exclusively with him. With a menu, each retailer can instead offer both a contract that works well if accepted together with a contract from the other retailer and a contract that works well if accepted on its own. Should either retailer demand too much of the surplus, the manufacturer can respond by accepting an attractive (i.e., profit-maximizing) option for dealing with only the other retailer.

The fact that a retailer can design a contract intended for an "exclusive" relationship in a way that ensures $M$ will not also accept a contract from the retailer's rival follows from the same logic as in Marx and Shaffer's paper: in the "exclusive" contract each retailer $R_{i}$ is indifferent about whether to purchase, so acceptance of a contract from the rival $R_{-i}$ causes $R_{i}$ to cease carrying $M$ 's goods, which makes accepting $R_{-i}$ 's contract unprofitable. As noted by Miklos-Thal et al., however, slotting fees are not essential for accomplishing this goal. For example, we show 
in the Appendix that when $M$ faces a constant unit cost $c$, under mild regularity conditions the retailers can sustain the monopoly outcome by offering menus consisting of the following two options, which coincide with the previous tariffs $T_{i}^{C}$ and $T_{i}^{E}$ for large enough quantities but involve no slotting fees and cover the manufacturer's costs regardless of the quantity purchased:

- an option designed for common agency, satisfying $\hat{T}_{i}^{C}\left(q_{i}\right)=c q_{i}$ for $q \leq q_{i}^{C}$, and $\hat{T}_{i}^{C}\left(q_{i}\right)=F_{i}^{C}+w_{i}^{*} q_{i}$ for $q>q_{i}^{C}$, where $q_{i}^{C}$ and $F_{i}^{C}$ are chosen so that, given $R_{-i}$ 's equilibrium behavior, $R_{i}$ can obtain its contribution $\Delta_{i}$ either by selling $q_{i}^{C}$ (and buying it at cost) or by selling the larger profit-maximizing quantity $q_{i}^{*} \equiv q_{i}\left(w_{i}^{*}, w_{-i}^{*}\right)$ [and paying $\left.\hat{T}_{i}^{C}\left(q_{i}^{*}\right)\right]$

- an option designed for exclusive dealing, $\hat{T}_{i}^{E}$, satisfying $\hat{T}_{i}^{E}\left(q_{i}\right)=$ $c q_{i}$ for $q \leq q_{i}^{E}$ and $\hat{T}_{i}^{E}\left(q_{i}\right)=F_{i}^{E}+w_{i}^{m} q_{i}$ for $q>q_{i}^{E}$, where $q_{i}^{E}$ and $F_{i}^{E}$ are chosen so that, absent $R_{-i}, R_{i}$ can obtain its contribution $\Delta_{i}$ by buying $q_{i}^{E}$ (at cost) as well as by buying the larger bilateral monopoly quantity $q_{i}^{m} \equiv q_{i}^{m}\left(w_{i}^{m}, \infty\right)$ [and paying $\left.\hat{T}_{i}^{E}\left(q_{i}^{m}\right)\right]$.

\section{Extensions}

Following Marx and Shaffer, we have focused so far on the set of outcomes achievable with contracts in which retailers face constant marginal wholesale prices. When retailer demand functions are not concave, this may limit the ability to support the industry profit-maximizing outcome. That is, letting $\rho_{i}\left(q_{i}, q_{-i}\right)$ denote the revenue generated by $R_{i}$ 's sales, in the model studied above it may be that $\Pi^{*}<\max _{q_{1}, q_{2}}$ $\sum_{i} \rho_{i}\left(q_{i}, q_{-i}\right) q_{i}-c\left(q_{1}, q_{2}\right)$. Here we show that similar insights apply to the unconstrained industry profits and, equally important, we extend the analysis to any number of retailers. 
Suppose that there are $n$ retailers, and let $N \equiv\{1, \ldots, n\}$. For any quantity profile $q \in Q=\left\{\left(q_{i}\right)_{i \in N} \mid q_{i} \in \mathbb{R}_{+}\right\}$, denote the industry profit by $\Pi(q) \equiv \sum_{i \in N} \rho_{i}(q)-c(q)$. Finally, for any subset of retailers $S \subset N$, define $Q^{S}=\left\{q \in Q \mid q_{i}=0\right.$ if $\left.i \notin S\right\}$ and suppose that the maximal profit that $M$ and the retailers in $S$ can obtain together, denoted $\Pi^{S} \equiv$ $\max _{q \in Q^{S}} \Pi(q)$, is achieved at $q=q^{S}$. These retailers' joint contribution to industry profit is then defined as $\Delta_{S} \equiv \Pi^{N}-\Pi^{N \backslash S}$. For the sake of exposition, we will use the notation $\Delta_{i} \equiv \Delta_{\{i\}}$ for $R_{i}$ 's individual contribution. As before, $\Delta_{M} \equiv \Pi^{N}-\sum_{i \in N} \Delta_{i}$ is $M$ 's residual profit. To reflect retailers' imperfect substitutability, we assume:

Assumption 1': For any subset $S$ such that $\# S \geq 2, \Delta_{S}>$ $\sum_{i \in S} \Delta_{i}$

Assumption 1' asserts that retailers are substitutes to each other in generating industry profit. For example, in the two retailer model studied above, $\Delta_{\{1,2\}}=\Pi^{*}$, so part (ii) of the assumption amounts to $\Pi^{*}>\sum_{i \in S}\left(\Pi^{*}-\Pi_{-i}^{m}\right)$, i.e., to $\sum_{i \in S} \Pi_{i}^{m}>\Pi^{*}$.

The retailers can again use menus of contracts to sustain the industry profit $\Pi^{N}$. To see this, suppose that each $R_{i}$ offers a contract consisting of the following options:

- a tariff $T_{i}^{C}$ designed for common agency, giving $R_{i}$ the choice between $C_{i}^{0} \equiv\left(-\Delta_{i}, 0\right)$, which consists of buying nothing and receiving a payment from $M$ equal to $\Delta_{i}$, or $C_{i}^{C} \equiv\left(F_{i}^{C}, q_{i}^{N}\right)$, which consists of buying $q_{i}^{N}$ in exchange for a payment $F_{i}^{C} \equiv \rho_{i}\left(q^{N}\right)-\Delta_{i}$;

- for each rival $R_{j}$, a tariff $T_{i j}^{E}$ designed for the case where $R_{j}$ is excluded, giving $R_{i}$ the choice between $C_{i}^{0}$ or $C_{i j}^{E} \equiv\left(F_{i j}^{E}, q_{i}^{N \backslash\{j\}}\right)$, which consists of buying $q_{i}^{N \backslash\{j\}}$ in exchange for a payment $F_{i j}^{E} \equiv$ $\rho_{i}\left(q^{N \backslash\{j\}}\right)-\Delta_{i}$ 
If all retailers offer these contracts and $M$ accepts the options $\left(T_{i}^{C}\right)_{i \in N}$, there is a continuation equilibrium in which each $R_{i}$ opts for $C_{i}^{C}$ (each $R_{i}$ is then indifferent between $C_{i}^{0}$ and $C_{i}^{C}$ ). This continuation equilibrium sustains the industry profit maximum $\Pi^{N}$, gives each $R_{i}$ its contribution $\Delta_{i}$, and thus gives $M$ its residual contribution $\Delta_{M}$. The joint profit of $M$ and any subset $S$ of retailers is moreover at least what they could obtain on their own since

$$
\Delta_{M}+\sum_{i \in S} \Delta_{i}=\Pi^{N}-\sum_{j \in N \backslash S} \Delta_{j} \geq \Pi^{N}-\Delta_{N \backslash S}=\Pi^{S}
$$

where the inequality follows from part (ii) of Assumption 1'. This, in turn, implies that $M$ cannot benefit from selecting any other set of options, since any $R_{i}$ whose offer is accepted can secure its contribution $\Delta_{i}$ by opting for $C_{i}^{0}$, and dealing with a set $S$ of retailers cannot generate more than $\Pi^{S}$.

Finally, no $R_{i}$ can benefit from deviating. To see this, observe first that $M$ can also secure its contribution $\Delta_{M}$ by accepting the tariffs $\left(T_{j i}^{E}\right)_{j \in N \backslash\{i\}}$ : this induces every other retailer $R_{j}, j \neq i$, to opt for $C_{j i}^{E}$ (this is indeed a continuation equilibrium since each $R_{j}$ is then indifferent between $C_{j}^{0}$ and $C_{j i}^{E}$ ) and thus gives $M$ a profit equal to

$$
\Pi^{N \backslash\{i\}}-\sum_{j \in N \backslash\{i\}} \Delta_{j}=\Pi^{N}-\left(\Pi^{N}-\Pi^{N \backslash\{i\}}\right)-\sum_{j \in N \backslash\{i\}} \Delta_{j}=\Pi^{N}-\sum_{j \in N} \Delta_{j}=\Delta_{M} .
$$

Moreover, any $R_{j}$ whose offer is accepted can again secure its contribution $\Delta_{j}$ by opting for $C_{j}^{0}$. Thus, if a deviation by $R_{i}$ leads $M$ to accept contracts from the retailers in subset $S$, then $R_{i}$ 's profit is bounded above 
by

$$
\begin{aligned}
\Pi^{S}-\Delta_{M}-\sum_{j \in S \backslash\{i\}} \Delta_{j} & =\left(\Pi^{N}-\Delta_{N \backslash S}\right)-\left(\Pi^{N}-\sum_{j \in N} \Delta_{j}\right)-\sum_{j \in S \backslash\{i\}} \Delta_{j} \\
& =\Delta_{i}+\sum_{j \in N \backslash S} \Delta_{j}-\Delta_{N \backslash S} \\
& \leq \Delta_{i},
\end{aligned}
$$

where the inequality follows again from Assumption 1'.

Alternatively, one could build on Segal (1999)'s result mentioned in the Introduction. To sustain the industry profit maximum, suppose that each retailer $R_{i}$ offers the manufacturer the following contract: "For a payment of $\Delta_{i}$ you can make a take-it-or-leave-it offer to me." If $M$ accepts all offers, then by Segal's result the industry profit-maximizing outcome arises, and each party receives the same payoff as above. Moreover, since $M$ and retailers in $S \subset N$ obtain $\Pi^{S}$ in the candidate equilibrium, $M$ is indeed willing to accept all the offers if they are made. Now consider whether any retailer has an incentive to deviate. The argument parallels that above: Any $R_{i}$ whose offer is accepted obtains its contribution $\Delta_{i}$ and, from (1), $M$ can also secure its own contribution $\Delta_{M}$ by accepting the offers of all of $R_{i}$ 's rivals. Therefore, by inducing $M$ to accept the offers of a subset $S$ of retailers, $R_{i}$ cannot earn more than $\Pi^{S}-\Delta_{M}-\sum_{j \in S \backslash\{i\}} \Delta_{j} \leq \Delta_{i}$. 


\section{Appendix}

We show in this Appendix that, when $M$ faces a constant marginal $\operatorname{cost} c$, it is possible to sustain the industry profit maximum $\Pi^{*}$ by offering the tariffs $\hat{T}_{i}^{C}$ and $\hat{T}_{i}^{E}$ described in the text. The exact specification of the options depends on the nature of retail competition. For the sake of exposition, we will assume here that: (i) retailers compete in quantities (a similar analysis applies to price competition); (ii) the revenue generated by $R_{i}$ 's sales, $\rho_{i}\left(q_{i}, q_{-i}\right)$, is continuous (with $\rho_{i}\left(0, q_{-i}\right)=0$ for all $q_{-i}$ ), strictly concave in $q_{i}$, decreasing in $q_{-i}$ (and strictly so if $\left.\rho_{i}\left(q_{i}, q_{-i}\right)>0\right)$, and leads quantities to be strategic substitutes, i.e., $\partial^{2} \rho_{i} / \partial q_{i} \partial q_{-i}<0$. Let $\left(q_{1}^{*}, q_{2}^{*}\right)$ denote the quantities purchased in the industry profit maximum supported by wholesale prices $\left(w_{1}^{*}, w_{2}^{*}\right)$, and let $q_{i}^{m}$ denote the quantity, supported by $w_{i}^{m}$, that maximizes profits in an exclusive relationship with $R_{i}$. Note that under these assumptions, $w_{i}^{m}=c .^{4}$

The parameters of the tariff $\hat{T}_{i}^{E}$ are designed so that $q_{i}^{E}$ is the smallest quantity satisfying $\rho_{i}\left(q_{i}, 0\right)-c q_{i}=\Delta_{i}$, and $F_{i}^{E}=\Pi_{i}^{m}-\Delta_{i}=\Delta_{M}$. Similarly, the parameters of the tariff $\hat{T}_{i}^{C}$ are designed so that $q_{i}^{C}$ is the smallest quantity satisfying $\rho_{i}\left(q_{i}, q_{-i}^{*}\right)-c q_{i}=\Delta_{i}$, and $F_{i}^{C}=\rho_{i}\left(q_{i}^{*}, q_{-i}^{*}\right)-$ $w_{i}^{*} q_{i}^{*}-\Delta_{i}$. It is straightforward to check that the quantity thresholds lie below the (industry or bilateral) monopoly levels (that is, $q_{i}^{C}<q_{i}^{*}$ and $\left.q_{i}^{E}<q_{i}^{m}\right){ }^{5}$ and that the tariffs jump upwards at these thresholds (so

\footnotetext{
${ }^{4}$ In addition, given the concavity assumption made here, the linear wholesale prices

$$
w_{i}^{*}=c+\left[\frac{\partial \rho_{-i}\left(q_{-i}^{*}, q_{i}^{*}\right)}{\partial q_{i}}\right] q_{-i}^{*} \text { for } i=1,2
$$

induce the true industry profit maximum $\max _{q_{1}, q_{2}} \sum_{i} \rho_{i}\left(q_{i}, q_{-i}\right) q_{i}-c\left(q_{1}+q_{2}\right)$.

${ }^{5}$ This follows from our concavity assumption since the quantity $q_{i}^{C}$ is the smallest
} 
payments exceed the manufacturer's cost at all quantities). ${ }^{6}$

By construction, if $M$ chooses $\hat{T}_{1}^{C}$ and $\hat{T}_{2}^{C}$, there exists a continuation equilibrium yielding the profit-maximizing outcome: each $R_{i}$, anticipating $q_{-i}=q_{-i}^{*}$, finds it optimal to choose $q_{i}^{*}$ in the range $q_{i}>q_{i}^{C}$ where it faces the wholesale price $w_{i}^{*}$, is by construction indifferent between $q_{i}^{*}$ and $q_{i}^{C}$, and prefers these quantities to any quantity $q_{i}<q_{i}^{C}$; it is thus willing to choose $q_{i}=q_{i}^{*}$. In this continuation equilibrium, each $R_{i}$ obtains his contribution to industry profits $\left(\Delta_{i}\right)$, whereas $M$ obtains $\Delta_{M}$ solution to $\rho_{i}\left(q_{i}, q_{-i}^{*}\right)-c q_{i}=\Delta_{i}$, where

$$
\begin{aligned}
\Delta_{i} & =\Pi^{*}-\Pi_{-i}^{m} \\
& =\rho_{i}\left(q_{i}^{*}, q_{-i}^{*}\right)-c q_{i}^{*}+\rho_{-i}\left(q_{-i}^{*}, q_{i}^{*}\right)-c q_{-i}^{*}-\left[\rho_{-i}\left(q_{-i}^{m}, 0\right)-c q_{-i}^{m}\right] \\
& <\rho_{i}\left(q_{i}^{*}, q_{-i}^{*}\right)-c q_{i}^{*},
\end{aligned}
$$

whereas $q_{i}^{E}$ is the smallest solution to $\rho_{i}\left(q_{i}, 0\right)-c q_{i}=\Delta_{i}$, where

$$
\Delta_{i}=\Pi^{*}-\Pi_{-i}^{m}<\Pi_{i}^{m}=\max _{q_{i}} \rho_{i}\left(q_{i}, 0\right)-c q_{i} .
$$

${ }^{6}$ This fact derives from Assumption 1 for $\hat{T}_{i}^{E}$, which, since $w_{i}^{m}=c$, jumps by $\Delta_{M}>0$ at $q_{i}^{E}$. For $\hat{T}_{i}^{C}$, the jump at $q_{i}^{C}$ is equal to

$$
J_{i} \equiv\left(w_{i}^{*}-c\right) q_{i}^{C}+F_{i}^{C},
$$

where $F_{i}^{C}$ is chosen so that:

$$
\rho_{i}\left(q_{i}^{*}, q_{-i}^{*}\right)-w_{i}^{*} q_{i}^{*}-F_{i}^{C}=\Delta_{i}=\rho_{i}\left(q_{i}^{C}, q_{-i}^{*}\right)-c q_{i}^{C} .
$$

We thus have:

$$
\begin{aligned}
J_{i} & =\left(w_{i}^{*}-c\right) q_{i}^{C}+\rho_{i}\left(q_{i}^{*}, q_{-i}^{*}\right)-w_{i}^{*} q_{i}^{*}-\left[\rho_{i}\left(q_{i}^{C}, q_{-i}^{*}\right)-c q_{i}^{C}\right] \\
& =\rho_{i}\left(q_{i}^{*}, q_{-i}^{*}\right)-w_{i}^{*} q_{i}^{*}-\left[\rho_{i}\left(q_{i}^{C}, q_{-i}^{*}\right)-w_{i}^{*} q_{i}^{C}\right] \\
& =\max _{q_{i}}\left\{\rho_{i}\left(q_{i}, q_{-i}^{*}\right)-w_{i}^{*} q_{i}\right\}-\left[\rho_{i}\left(q_{i}^{C}, q_{-i}^{*}\right)-w_{i}^{*} q_{i}^{C}\right]>0,
\end{aligned}
$$

where the last equality derives from the fact that $\left(q_{1}^{*}, q_{2}^{*}\right)$ is a Nash equilibrium given wholesale prices $\left(w_{1}^{*}, w_{2}^{*}\right)$ and the inequality stems from $q_{i}^{C} \neq q_{i}^{*}$ and strict concavity. 
$\left(=\Pi^{*}-\Delta_{1}-\Delta_{2}\right)$; the joint profit of $M$ and each retailer $R_{i}, \Delta_{i}+\Delta_{M}$, thus equals $\Pi_{i}^{m}$.

If the retailers make the above-described offers, $M$ cannot gain from accepting any other combination of options: $M$ can obtain $\Delta_{M}$ by accepting only $\hat{T}_{i}^{E}$ since, anticipating being alone in the market, $R_{i}$ is willing to buy $q_{i}^{m}$, which gives $\Delta_{M}$ to $M$ and $\Delta_{i}$ to $R_{i}$. If instead $M$ accepts only $\hat{T}_{i}^{C}$, then $R_{i}$ can secure more than $\Delta_{i}$ (e.g., by choosing $\left.q_{i}^{*}\right)$, since it no longer faces competition, implying that $M$ cannot obtain more than $\Pi_{i}^{m}-\Delta_{i}=\Delta_{M}$. If $M$ instead accepts $\hat{T}_{1}^{E}$ and $\hat{T}_{2}^{E}$, then from strategic substitutability either each $R_{i}$ chooses $q_{i} \leq q_{i}^{E}$ and $M$ obtains zero profit, or one retailer chooses $q_{i}^{m}$ while its rival chooses $q_{-i}=0$, and $M$ obtains again $\Delta_{M}$. Finally, suppose that $M$ accepts $\hat{T}_{i}^{E}$ and $\hat{T}_{-i}^{C}$. If $R_{-i}$ is not active, then again $R_{i}$ chooses $q_{i}^{m}$ and $M$ obtains $\Delta_{M}$. If $R_{-i}$ is active (i.e., $q_{-i}>0$ ), then from strategic substitutability $R_{i}$ chooses again $q_{i} \leq q_{i}^{E}$ and appropriates all the profit generated by its sales. Furthermore, if $q_{i}>q_{i}^{*}$ then $R_{-i}$ chooses $q_{-i} \leq q_{-i}^{C}$ and $M$ obtains zero profit; if instead $q_{i} \leq q_{i}^{*}$, then $R_{-i}$ obtains at least $\Delta_{i}$ (e.g., by choosing $\left.q_{-i}^{*}\right)$ and thus, since its sales cannot generate more than $\Pi_{i}^{m}, M$ cannot obtain more than $\Pi_{i}^{m}-\Delta_{i}=\Delta_{M}$.

Summing-up, if the retailers offer the above-mentioned options, there is a continuation equilibrium in which $M$ chooses $\hat{T}_{1}^{C}$ and $\hat{T}_{2}^{C}$, and then the retailers implement the profit-maximizing outcome. We now check that no retailer has an incentive to deviate.

As before, a deviation by $R_{-i}$, say, can be profitable only if it increases the joint profit of $M$ and $R_{-i}$ above the equilibrium level, $\Pi_{-i}^{m}$, since $M$ can always secure $\Delta_{M}$ by accepting $\hat{T}_{i}^{E}$ only. It follows that $R_{-i}$ cannot benefit from deviating in a way that leads $M$ to reject $R_{i}$ 's offers, since they cannot generate more than $\Pi_{-i}^{m}$ in this way. 
Consider now a deviation by $R_{-i}$ that leads $M$ to accept $\hat{T}_{i}^{E}$. Then, as above, either $R_{-i}$ ends up selling nothing, in which case $R_{i}$ can secure $\Delta_{i}$ (e.g., by choosing $q_{i}=q_{i}^{m}$ ) and thus the joint profit of $M$ and $R_{-i}$ cannot exceed $\Pi^{*}-\Delta_{i}=\Pi_{-i}^{m}$, or because $R_{-i}$ sells a positive amount, $R_{i}$ will buy less than $q_{i}^{E}$, in which case $M$ supplies $R_{i}$ at cost, and thus the joint profit of $M$ and $R_{-i}$ equals $\rho_{-i}\left(q_{-i}, q_{i}\right)-c q_{-i}$, which cannot exceed $\Pi_{-i}^{m}=\max _{q_{-i}}\left[\rho_{-i}\left(q_{-i}, 0\right)-c q_{-i}\right]$.

Finally, consider a deviation by $R_{-i}$ that leads $M$ to accept $\hat{T}_{i}^{C}$. If $R_{-i}$ ends up selling more than $q_{-i}^{*}$, then $R_{i}$ will buy - at cost - less than $q_{i}^{C}$; the joint profit of $M$ and $R_{-i}$ then again equals $\left[\rho_{-i}\left(q_{-i}, q_{i}\right)-c q_{-i}\right] \leq$ $\Pi_{-i}^{m}$. If instead $R_{-i}$ ends up selling less than $q_{-i}^{*}$, then $R_{i}$ can secure $\Delta_{i}$ (e.g., by choosing $q_{i}=q_{i}^{m}$ ) and thus, again, the joint profit of $M$ and $R_{-i}$ cannot exceed $\Pi^{*}-\Delta_{i}=\Pi_{-i}^{m}$. 


\section{References}

\section{References}

[1] Martimort, D. and L. Stole (2003), "Contractual Externalities and Common Agency Equilibria," Advances in Theoretical Economics 3: Article 4.

[2] Marx,. L. and G. Shaffer (2007), "Upfront Payments and Exclusion in Downstream Markets," RAND Journal of Economics 38: 823-43.

[3] Miklos-Thal, J., P. Rey, and T. Verge (forth.), "Buyer Power and Intrabrand Competition," Journal of the European Economic Association.

[4] Segal, I. (1999), "Contracting with Externalities," Quarterly Journal of Economics 114: 337-88.

[5] Segal, I. and M.D. Whinston (2003), "Robust Predictions for Contracting with Externalities," Econometrica 71: 757-91. 